\section{Noticias y comentarios}

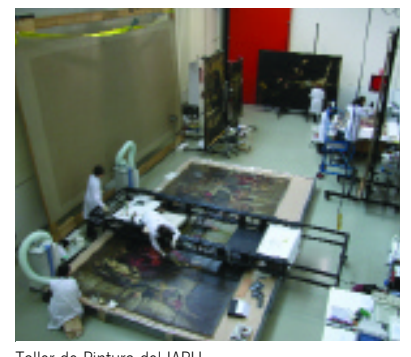

Taller de Pintura del IAPH

\title{
El Taller de Pintura presenta su experiencia en reentelado de gran formato
}

En el marco del $1^{\text {er }}$ Seminario Internacional de Pintura (XVI Congreso Internacional de Conservación y Restauración de Bienes Culturales del Grupo Español del ICOM e Instituto de Restauración de Patrimonio de la Universidad Politécnica de Valencia), técnicos del Centro de Intervención del Instituto Andaluz del Patrimonio Histórico presentaron el proyecto "Reentelado de grandes formatos. Actualización de métodos tradicionales: Metodología de actuación". El Seminario, organizado bajo el subtítulo "El soporte textil: comportamiento, deterioro y criterios de intervención" por la UPV (9 al 11 de marzo de 2005), contó con la participación de un nutrido grupo de investigadores de reconocido prestigio de Italia, Inglaterra, Francia, Estados Unidos y España, que presentaron los últimos estudios y experiencias en materia de conservación y restauración de pintura sobre lienzo, analizando su comportamiento, deterioro y criterios de actuación.

El Taller de Pintura del Departamento de Tratamiento del IAPH ha llevado a cabo numerosos proyectos de intervención de pinturas de gran formato sobre soporte de lienzo. Obras de grandes proporciones han sido incluidas en las programaciones anuales y han formado parte de los proyectos especiales de actuación sobre grandes colecciones o exposiciones: exposición "Velázquez y Sevilla", proyectos Palacio de San Telmo e Iglesia del Salvador, etc.

Las características técnicas de las instalaciones, la infraestructura y el espacio del Taller de Pintura lo hacen idóneo para abarcar varios trabajos de restauración de forma simultánea y facilitan el trabajo y movilidad de los equipos de técnicos necesarios para la realización de la fase de reentelado. Estos factores fundamentaron en parte el contenido de la ponencia, sobre experiencias obtenidas en los proyectos más singulares.

Como introducción al planteamiento técnico de la conferencia se mostraron en primer lugar las características metodológicas y los criterios de actuación del Centro de Intervención en las que se basan todos los proyectos que el Departamento de Tratamiento desarrolla. Seguidamente se trató esencialmente el uso actual de los métodos tradicionales de reentelado en instalaciones con infraestructuras modernas. Para ello se tomaron como ejemplo tres proyectos de intervención sobre obras de gran formato que presentan por un lado características técnicas muy diferentes en cuanto a época de ejecución, medidas o deterioros y por otro, rasgos comunes como materialidad y alteraciones. Teniendo en cuenta estas características técnicas los tratamientos se han desarrollado utilizando unos materiales u otros según los casos pero teniendo como finalidad común una actuación de consolidación de soporte (reentelado) utilizando el método de trabajo tradicional.

Amalia Cansino Cansino

$M^{\mathrm{a}}$ del Mar González González

Centro de Intervención del IAPH

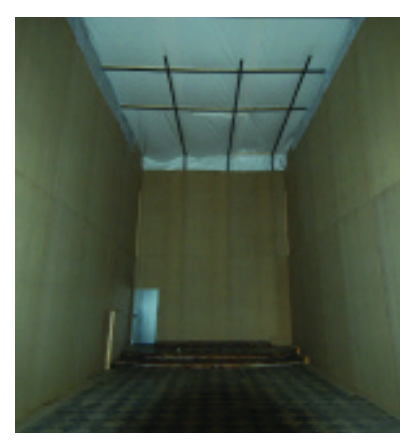

Capilla de San Telmo. Imagen de la protección de los retablos y de la bóveda

\section{San Telmo: traslado de bienes muebles y protección de retablos}

La Consejería de Cultura a través del Instituto Andaluz de Patrimonio Histórico ha elaborado el proyecto de conservación-restauración de la colección de bienes muebles de la Capilla del Palacio de San Telmo (Sevilla), en el que interviene un amplio equipo interdisciplinar especializado en las distintas tipologias de los bienes muebles de la colección objeto de estudio, así como de los retablos y las pinturas murales que decoran la capilla.

La ejecución del proyecto se llevará a cabo en tres años (2005-2008), de acuerdo al programa metodológico del IAPH.

Una vez concluida la primera fase previa a la intervención en la capilla, que consistió en el desmontaje, desinsectación y embalaje de las obras seleccionadas, se procedió al traslado de las mismas a las instalaciones del Instituto Andaluz de Patrimonio Histórico, donde previamente se han acondicionado diversas dependencias para su almacenaje.

Acto seguido al traslado se acometieron los trabajos de protección de la capilla, cuya actuación se ha centrado en los cinco retablos, la cúpula del cancel, los miradores con celosías, todo el zócalo de pintura mural de la capilla y la azulejería existente en la zona de acceso al camerín del Retablo Mayor.

También se han protegido dos marcos de gran formato, que tuvieron que ser desmontados en el transcurso de la fase previa de intervención. 


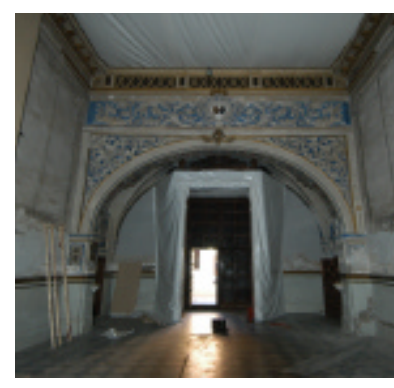

Capilla de San Telmo. Cancel de entrada tras la protección
Esta protección, necesaria antes de proceder a cualquier tipo de intervención en el edificio, ha consistido en:

$>$ Un complejo sistema de anclajes, mediante la elaboración de una liviana estructura portante consistente en listones de madera de conífera sujetos mediante espirros de impacto ubicados sobre la cornisa corrida, justo en zonas del muro exentas de ornamentación (molduras, pinturas...). Esta instalación sostiene, a modo de colgadura, una primera protección flexible a base de una capa de "tejido no tejido", y una segunda barrera de lona ignifuga e hidrófuga. De este modo, se evita el contacto directo sobre cualquier pieza y los efectos negativos del sobrepeso.

$>$ El espacio abierto entre la estructura descrita y los muros perimetrales se cubren, techándolos con tableros de madera, para impedir que caigan sobre el inmueble los nuevos aportes de polvo, suciedad y escombros de la obra (intervención de cubierta).

$>$ El espacio intermedio creado por la estructura metálica se cubre con una loneta que apoya en el entramado horizontal, con la finalidad de evi- tar el ascenso de depósitos de suciedad por la obra en la solería, que afectaría gravemente tanto a los elementos protegidos como a la pintura mural de la bóveda.

Con esta actuación se podrá garantizar la adecuada ventilación del ámbito intervenido así como la permanencia y estabilidad de los materiales durante el periodo solicitado.

Una vez finalizada esta actuación se procederá a la intervención arquitectónica prevista en la iglesia, que abarcará un periodo de dieciocho meses, tras lo cual se desmontarán los sistemas de protección permitiendo de este modo el comienzo de la intervención in situ de los bienes allí ubicados.

Pedro Castillo Pérez

Araceli Montero Moreno

Centro de Intervención del IAPH

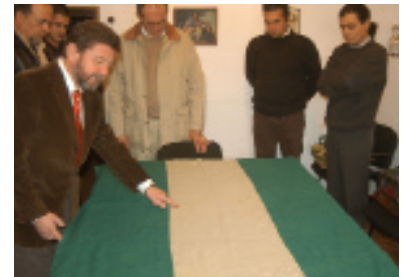

El Director General de Bienes Culturales, Jesús Romero, y técnicos del IAPH supervisan el estado de conservación de la Bandera de Andalucia en la casa Museo de Blas Infante

\section{Intervención en la Bandera de Andalucía expuesta en la casa Museo de Blas Infante}

El Instituto Andaluz de Patrimonio Histórico está acometiendo la intervención de la Bandera de Andalucía de Blas Infante, propiedad de la Junta de Andalucía, que se encuentra expuesta en la casa Museo de Blas Infante en Coria del Río, Sevilla, junto con otras piezas de la misma relevancia simbólica.

El carácter emblemático de la obra junto su situación actual ha agravado su estado de conservación debido a que se guarda plegada en exceso en una urna de cristal, de reducidas dimensiones, provocando el debilitamiento de la fibra y consecuentes roturas. La importancia simbólica y el estado de conservación han llevado a la restauración de la obra y de su contenedor.

El día 3 de noviembre de 2004 se efectuó el traslado de la Bandera por técnicos del Centro de Intervención del IAPH a las instalaciones de la institución. En el embalaje se empleó un contenedor especial que permitía el transporte con la mayor de las garantías.

Una vez ubicada la pieza en el taller de tejidos se ha realizado un examen preliminar exhaustivo para determinar su estado de conservación y tratamiento de intervención. La obra presenta fragilidad de la tela con la presencia de numerosos desgastes de fibras, desgarros, lagunas, así como manchas de diversas características y suciedad generalizada.

Paralelamente al estudio técnico realizado en el taller se han efectuado, en colaboración con la Policía Científica de Sevilla, pruebas para la identificación de ciertas manchas en la tela.

La intervención realizada se ha basado en tres fases fundamentales:

$>$ Limpieza con agua desmineralizada del tejido, tras comprobar la estabilidad al medio acuoso de los tintes.

$>$ Eliminación de intervenciones del tipo de zurcidos, que estaban provocando tensiones en la tela que podrian llegar a romperla.

$>$ Consolidación de las lagunas, roturas y zonas debilitadas por desgaste mediante la fijación a soportes locales situados en el reverso.

Actualmente se está estudiando el sistema expositivo que se adecue más a la pieza y a su ubicación en las dependencias de la Casa-Museo. 